\title{
Determination of parameters for Cauchy's problem for systems of ODEs with application to biological modelling
}

\author{
A.N. Pete*, P. Mathye*, I. Fedotov* and M. Shatalov*† \\ * Department of Mathematics and Statistics \\ Tshwane University of Technology, Pretoria, South Africa \\ mathyeph@gmail.com; FedotovI@tut.ac.za; ShatalovM@tut.ac.za \\ ${ }^{\dagger}$ Manufacturing and Materials \\ Council for Scientific and Industrial Research (CSIR), Pretoria, South Africa
}

Received: 13 March 2015, accepted: 23 April 2016, published: 9 May 2016

\begin{abstract}
An inverse numerical method that estimates parameters of dynamic mathematical models given some information about unknown trajectories at some time is applied to examples taken from Biology and Ecology. The method consists of determining an overdetermined system of algebraic equations using the experimental data. The solution of the overdetermined system is then obtained using, for example the least-square method. To illustrate the effectiveness of the method an analysis of examples and a numerical example for the model that monitors the dynamics of HIV is presented.
\end{abstract}

Keywords-Inverse problem; least squares methods; parameter estimation; dymamic systems; predator-prey system;

\section{INTRODUCTION}

Function approximation on a fixed interval by means of an initial value problem of an ordinary differential equation with unknown coefficients and unknown initial values as presented by $\mathrm{M}$ Shatalov, I. Fedotov and S.V. Joubert in [7], is central to this study. In their paper al method to determine both the unknown coefficients and initial values of a dynamic system by minimizing a certain goal function is presented. In earlier collaborations Shatalov and Fedotov suggested the use of such an approach in identifying dynamic systems' parameters from experimental data, see [6].

Several other parameter estimation methods are presented in literature, for instance the stochastic models; the Bayesion approach, the Monte Carlo technique, the numerical method with combined Adomain/Alienor approach, the differential evolution (DE) and the hybrid Taguchi-differential evolution algorithm.

The method used is based on integrating both sides of equations of a dynamic system, and applying regression methods to the overdetermined system of linear algebraic equations with possible constraints. The unknown parameters and initial values can then be obtained using the method of least squares. In this paper, the proposed method gives parameter estimates that have a percentage relative error that is mostly less than $0.4 \%$ for artificially generated data and parameters that are in the expected range for real data.

As an illustration of the method of identifica- 
tion, we present the analysis for problems from Biology and Ecology. The following mathematical models are taken as examples: model of a free population (with negligible mortality and with mortality different from zero), population with negligible mortality and unknown initial conditions, interspecies modifications of the Lotka-Voltera model and a model that monitors the dynamics of HIV. Numerical results with artificially generated data and real data is then given for the model that monitors the dynamics of HIV.

\section{A. Inverse methods}

The general approach to the inverse system identification of a $n$-dimensional parameter $\mathbf{a} \in$ $\mathcal{A} \subset \mathbb{R}^{n}$, where $\mathcal{A}$ can coincide with $\mathbb{R}^{n}$ (no constrains between entries of a) and $\mathcal{A}$ can be subset of $\mathbb{R}^{n}$ (there are constrains) in a system of ordinary differential equations of the following form

$$
\dot{\mathbf{x}}=\mathbf{f}(t, \mathbf{x}, \mathbf{a}),
$$

where $\mathbf{x}$ is vector-function $[0, T] \ni t \longrightarrow \mathbf{x}(t) \in$ $\mathbb{R}^{m}$ subject to experimental information concerning the values $\mathbf{x}\left(t_{j}\right)$ at the point $t_{j} \in[0, T]$ is known:

\begin{tabular}{|c|l|l|l|l|}
\hline$t_{0}$ & $\cdots$ & $t_{j}$ & $\cdots$ & $t_{N}$ \\
\hline $\mathbf{x}_{0}=\mathbf{x}\left(t_{0}\right)$ & $\cdots$ & $\mathbf{x}_{j}=\mathbf{x}\left(t_{j}\right)$ & $\cdots$ & $\mathbf{x}_{N}=\mathbf{x}\left(t_{N}\right)$ \\
\hline
\end{tabular}

The general approach to find a solution of the formulated problem (1) consists of determining an overdetermined system of algebraic equations using the experimental data (2)

$$
\mathbb{A} \mathbf{a}=\mathbf{h},
$$

with respect to unknown vector a. The solution of the system (3) is then obtained using any method of solution of the overdetermined system, for example the least-square method which minimize the difference $\mathbb{A} \mathbf{a}-\mathbf{h}$ using Euclidean metric. It is known that in this case (see, for example [5]), the solution of $(3)$ can be obtained by solving the following system

$$
\mathbb{A}^{\top} \mathbb{A} \mathbf{a}=\mathbb{A}^{\top} \mathbf{h}
$$

to obtain $\mathbf{a}$.

\section{MATHEMATICAL MODELS}

1) Problem 1: Free population: Consider a single species that grows by sexual reproduction. Assume that the individuals move in the population like Brownian motion particles (or that the population is colonial), then the frequency of contact between the individuals is proportional to the squared population density [1]. Further assuming that mortality is different from zero and is independent of the population size, the scalar function $\mathbf{f}(t, x, \mathbf{a})$ is given by

$$
\mathbf{f}(t, x, \mathbf{a})=\frac{a_{1} x^{2}}{x+a_{2}}-a_{3} x
$$

with the unknown vector $\mathbf{a}=\left(a_{1}, a_{2}, a_{3}\right)^{\top} \in \mathbb{R}^{3}$, $n=3, m=1$ and $a_{1}>a_{3}$. The parameters $a_{1}$ and $a_{2}$ represent per capita birth rate (fecundity) and the population at which half of the females are able to reproduce, respectively. The mortality rate of the population is represented by $a_{3}$. The parameters $a_{1}, a_{2}$ and $a_{3}$ are positive and $\mathbf{x}(t)=x(t) \in \mathbb{R}$ is a scalar function. We also assume that $x_{0}$ is specified.

A special case arises if we assume that the population is of negligible mortality. That is, if $a_{3}=0$ the scalar function $\mathbf{f}(t, x, \mathbf{a})$ is then given as

$$
\mathbf{f}(t, x, \mathbf{a})=\frac{a_{1} x^{2}}{x+a_{2}} .
$$

2) Problem 2: Population with negligible mortality and $x_{0}$ unknown: Here we consider the special case of Problem 1 (that is, if $a_{3}=0$ ) but with the value $x_{0}$ considered as an incorrect value which must be corrected. The statement of such a problem naturally appears since the initial value $x_{0}=x\left(t_{0}\right)$ plays an important role, namely; it defines the Cauchy's problem for equation (1) and, secondly $x_{0}$ is included widely in computations below. Therefore if $x_{0}$ given from an experiment has low accuracy, it would be desirable to define this value with more accuracy.

3) Problem 3:Nonlinear predation at small prey population: In this problem we consider an interspecies modification of the Lotka-Voltera model 
where there is a nonlinear predation at small prey population. Consider the system

$$
\left.\begin{array}{c}
\dot{x}=-a_{1} \frac{x^{2} y}{x^{p}+a_{2}}+a_{3} x, \\
\dot{y}=a_{4} \frac{x^{2} y}{x^{p}+a_{2}}-a_{5} y,
\end{array}\right\}
$$

where $p=1,2, a_{1}, a_{2}, a_{4}>0, a_{3}, a_{5} \geq 0$ and $n=5, m=2$. The prey and predator densities are represented by $x(t)$ and $y(t)$, respectively. In this model, the parameters $a_{1}$ represent the rate of the consumption of prey by the predator population, $a_{2}$ the prey population density at which the predator's consumption is half the maximum value [1], $a_{3}$ the prey's growth rate, $a_{4}$ is rate at which the prey contributes to the predator's growth rate and $a_{5}$ is the predator's death rate.

4) Problem 4: A model that monitors the $d y$ namics of HIV: Consider the following twodimensional model that monitors the dynamics of HIV. The model considers two sub-populations: HIV susceptible $(x)$, the HIV infected population (y). The total population size is given by $N=$ $x+y$. The model is described by

$$
\left.\begin{array}{l}
\dot{x}=-a_{1} \frac{x y}{N}-a_{2} x+a_{3}, \\
\dot{y}=a_{1} \frac{x y}{N}-a_{4} y,
\end{array}\right\}
$$

where $a_{1}, a_{2}, a_{3}$ and $a_{4}$ are all positive constants model parameters. The parameter $a_{1}$ and $a_{2}$ denotes the average rate of infection by HIV, $a_{2}$ the natural cessation of sexual activity, $a_{3}$ the recruitment rate of susceptible and $a_{4}$ denotes the death rates of the infected population due to HIV. This model is a modified version of the three dimensional one by Gumel (see, [3]). The vector $\mathbf{a}=\left(a_{1}, a_{2}, a_{3}, a_{4}\right)^{\top} \in \mathbb{R}^{4}$ is unknown. Note that in this case $n=4$ and $m=2$.

\section{Construction OF OVERDETERMined SYSTEMS}

Consider equation (1) where $\mathbf{f}$ is defined by

$$
\mathbf{f}(t, x, \mathbf{a})=\frac{a_{1} x^{2}}{x+a_{2}},
$$

The resultant equation can be rewritten as

$$
\dot{x} x+a_{2} \dot{x}=a_{1} x^{2},
$$

Integration of (10) with respect to $t$ from $t_{0}$ to $t_{j}$ $(j=1,2, \ldots, N)$ gives

$$
-a_{1} P_{j}+a_{2} \Delta x_{j}=-\Delta h_{j}
$$

where

$$
\left.\begin{array}{rl}
\Delta x_{j} & =x\left(t_{j}\right)-x\left(t_{0}\right), \\
P_{j} & =\int_{t_{0}}^{t_{j}} x^{2} d t, \\
\Delta h_{j} & =\left[\frac{1}{2} x^{2}\left(t_{j}\right)-\frac{1}{2} x^{2}\left(t_{0}\right)\right] \cdot
\end{array}\right\}
$$

The integral $P_{j}$ can be calculated by using a quadrature rule, for example trapezoidal rule. Thus, System (3) is solved with

$$
\begin{aligned}
& \mathbb{A}=\left(\begin{array}{cc}
-P_{1} & \Delta x_{1} \\
\vdots & \vdots \\
-P_{N} & \Delta x_{N}
\end{array}\right), \\
& \mathbf{a}=\left(\begin{array}{c}
a_{1} \\
a_{2}
\end{array}\right) \text { and } \mathbf{h}=\left(\begin{array}{c}
\Delta h_{1} \\
\vdots \\
\Delta h_{N}
\end{array}\right) .
\end{aligned}
$$

Now, suppose that (1) is defined as in (10) and the initial condition is unknown. Let us replace in (11) $\Delta x_{j}$ and $\Delta h_{j}$ by those given in (12):

$$
-a_{1} P_{j}+a_{2} x_{j}-\left(a_{2} x_{0}+\frac{1}{2} x_{0}^{2}\right)=-h_{j},
$$

where $h_{j}=\frac{1}{2} x_{j}^{2}$ under the assumption that for $P_{j}$ we use the old values of $x_{0}$ defined by (2) for $j=$ 1 and for $j \geq 2$ the values of $P_{j}$ are evaluated by an open quadrature rule. Formally speaking system (13) is nonlinear but setting

$$
-\left(a_{2} x_{0}+\frac{1}{2} x_{0}^{2}\right)=a_{3},
$$

we obtain the linear system of the form (3) with

$$
\begin{aligned}
& \mathbb{A}=\left(\begin{array}{ccc}
-P_{1} & x_{1} & 1 \\
\vdots & \vdots & \vdots \\
-P_{N} & x_{N} & 1
\end{array}\right), \\
& \mathbf{a}=\left(\begin{array}{c}
a_{1} \\
a_{2} \\
a_{3}
\end{array}\right), h=\left(\begin{array}{c}
-h_{1} \\
\vdots \\
-h_{N}
\end{array}\right) .
\end{aligned}
$$


A.N. Pete et al., Determination of parameters for Cauchy's problem ...

The value of $x_{0}$ can then be obtained from Equation (14).

Equation (1) with $\mathbf{f}$ defined by (5) can be written in the form

$$
x \dot{x}+a_{2} \dot{x}=a_{1} x^{2}-a_{3} x^{2}-a_{2} a_{3} x,
$$

In contrast to 10$)$ two supplementary terms $-a_{3} x^{2}$ and $-a_{2} a_{3} x$ arise. Integrating 15 we obtain

$$
\begin{aligned}
\left(a_{1}-a_{3}\right) P_{j}-a_{2} x_{j}+ & \left(\frac{1}{2} x_{0}^{2}+a_{2} x_{0}\right) \\
& -a_{2} a_{3} X_{j}=h_{j},
\end{aligned}
$$

where $X_{j}=\int_{t_{0}}^{t_{j}} x d t$. Introducing the new vector $\mathbf{b}=\left(b_{1}, b_{2}, b_{3}, b_{4}\right)$ where $b_{1}=a_{1}-a_{2}, b_{2}=a_{2}$, $b_{3}=\frac{1}{2} x_{0}^{2}+a_{2} x_{0}$ and $b_{4}=a_{2} a_{3}$ the system can be written in the form (3) where

$$
\begin{aligned}
\mathbb{A} & =\left(\begin{array}{cccc}
P_{1} & -x_{1} & 1 & -X_{1} \\
\vdots & \vdots & \vdots & \vdots \\
P_{N} & -x_{N} & 1 & -X_{N}
\end{array}\right), \\
\mathbf{b} & =\left(\begin{array}{c}
b_{1} \\
b_{2} \\
b_{3} \\
b_{4}
\end{array}\right) \text { and } h=\left(\begin{array}{c}
h_{1} \\
\vdots \\
h_{N}
\end{array}\right)
\end{aligned}
$$

In other words, we obtain the following system

$$
b_{1} P_{j}-b_{2} x_{j}+b_{3}-b_{4} X_{j}=h_{j},(j=1, \ldots N)
$$

Since the Jacobian is $\frac{\partial b}{\partial a}=a_{2}+x_{0} \neq 0$, we can find the vector $\mathbf{b}$ and hence the unknown vector a.

The system (7) of an interspecies modification of the Lotka-Voltera model for nonlinear predation at small prey population can be written as

$$
\left.\begin{array}{c}
\dot{x} x^{p}+a_{2} \dot{x}=-a_{1} x^{2} y+a_{3} x^{p+1}+a_{2} a_{3} x \\
\dot{y} x^{p}+a_{2} \dot{y}=a_{4} x^{2} y-a_{5} y x^{p}-a_{2} a_{5} y
\end{array}\right\}
$$

where $p=1,2, a_{1}, a_{2}, a_{4}>0$ and $a_{3}, a_{5} \geq 0$. Integrating (18) we obtain

$$
\left.\begin{array}{c}
\Delta h_{j}=-a_{1} Z_{j}-a_{2} \Delta x_{j}+a_{3} P_{j}+a_{2} a_{3} X_{j} \\
S_{j}=-a_{2} \Delta y_{j}+a_{4} Z_{j}-a_{5} Q_{j}-a_{2} a_{5} Y_{j}
\end{array}\right\}
$$

where,using the same notation as in (12):

$$
\left.\begin{array}{rl}
\Delta x_{j} & =x\left(t_{j}\right)-x\left(t_{0}\right), \\
P_{j} & =\int_{t_{0}}^{t_{j}} x^{2} d t, \\
\Delta h_{j} & =\left[\frac{1}{2} x^{2}\left(t_{j}\right)-\frac{1}{2} x^{2}\left(t_{0}\right)\right] \cdot
\end{array}\right\}
$$

and

$\begin{aligned} X_{j} & =\int_{t_{0}}^{t_{j}} x(t) d t, Y_{j}=\int_{t_{0}}^{t_{j}} y(t) d t \\ Z_{j} & =\int_{t_{0}}^{t_{j}} y(t) x^{2}(t) d t, Q_{j}=\int_{t_{0}}^{t_{j}} x^{p}(t) y(t) d t,\end{aligned}$

$S_{j}=\int_{t_{0}}^{t_{j}} x^{p}(t) d t$,

with $j=1 \ldots N$. Introducing the new vector $\mathbf{b}=$ $\left(b_{1}, b_{2}, b_{3}, b_{4}, b_{5}, b_{6}, b_{7}\right)^{\top}$ where $b_{1}=a_{1}, b_{2}=a_{2}$, $b_{3}=a_{3}, b_{4}=a_{4}, b_{5}=a_{5}, b_{6}=a_{2} a_{3}$ and $b_{7}=$ $a_{2} a_{5}$, we write the System (19) in the form (3) where

$\mathbb{A}=\left(\begin{array}{ccccccc}-Z_{1} & -\Delta x_{1} & P_{1} & 0 & 0 & X_{1} & 0 \\ -Z_{2} & -\Delta x_{2} & P_{2} & 0 & 0 & X_{2} & 0 \\ \vdots & \vdots & \vdots & \vdots & \vdots & \vdots & \vdots \\ -Z_{N} & -\Delta x_{N} & P_{N} & 0 & 0 & X_{N} & 0 \\ 0 & -\Delta y_{1} & 0 & Z_{1} & -Q_{1} & 0 & -Y_{1} \\ 0 & -\Delta y_{2} & 0 & Z_{2} & -Q_{2} & 0 & -Y_{2} \\ \vdots & \vdots & \vdots & \vdots & \vdots & \vdots & \vdots \\ 0 & -\Delta y_{N} & 0 & Z_{N} & -Q_{N} & 0 & -Y_{N}\end{array}\right)$

and

$$
\mathbf{h}=\left(\Delta h_{1}, \ldots, \Delta h_{N}, S_{1}, \ldots, S_{N}\right) .
$$

In other words, we obtain the following system of $2 N$ equations with seven unknowns:

$$
\left.\begin{array}{c}
\Delta h_{j}=-b_{1} Z_{j}-b_{2} \Delta x_{j}+b_{3} P_{j}+b_{6} X_{j}, \\
S_{j}=-b_{2} \Delta y_{j}+b_{4} Z_{j}-b_{5} Q_{j}-b_{7} Y_{j},
\end{array}\right\}
$$

subject to

$$
\left.\begin{array}{l}
-b_{6}+b_{2} b_{3}=0, \\
-b_{7}+b_{2} b_{5}=0 .
\end{array}\right\}
$$

To evaluate numerically the right hand side of $S_{j}$ can be considered as the Riemann-Stietjes integral $S_{j}=\int_{0}^{t_{j}} x^{p}(t) d y(t)$, (see, for example Dragomir and Fedotov [3]). Otherwise this integral can be computed using numerical differentiation. 
Another approach to construct the over determined system is generally appealing since the system obtained is simpler than (19). This method is possible due to the special form of the system (7); namely the system can be written as

$$
\left.\begin{array}{c}
\dot{x}=-a_{1} g\left(x, y, a_{2}\right)+a_{3} x, \\
\dot{y}=a_{4} g\left(x, y, a_{2}\right)-a_{5} y,
\end{array}\right\}
$$

where $g\left(x, y, a_{2}\right)=\frac{x^{2} y}{x^{p}+a_{2}}$. Eliminating $g\left(x, y, a_{2}\right)$ we get

$$
\dot{y}+c_{1} y+c_{2} \dot{x}-c_{3} x=0
$$

where

$$
a_{5}=c_{1}, \frac{a_{4}}{a_{1}}=c_{2} \text { and } \frac{a_{4} a_{3}}{a_{1}}=c_{3} .
$$

Using the integration techniques that we discussed earlier, we determine the vector $\mathbf{c}=\left(c_{1}, c_{2}, c_{3}\right)^{\top}$ and hence $\frac{c_{3}}{c_{2}}=a_{3}$, which will be considered as unknown in the next step.

Multiplying the first equation of (7) by $\left(x^{p}+a_{2}\right)$, we obtain

$$
x^{p} \dot{x}+a_{2} \dot{x}=-a_{1} x^{2} y+a_{3} x^{p+1}+a_{2} a_{3} x .
$$

After integration of 28 the overdetermined system can be written as

$$
a_{1} Z_{j}+a_{2}\left(\Delta x_{j}-a_{3} X_{j}\right)=-\Delta h_{j}+a_{3} P_{j},
$$

where $j=1, \ldots, N$, and $Z_{j}, P_{j}, X_{j}, \Delta h_{j}$, $\Delta x_{j}$ were defined in (20) and (22). The unknown parameters $a_{1}$ and $a_{2}$ may then be determined from equation (29) and $a_{4}$ and $a_{5}$ from (27).

\section{A. A model that monitors the dynamics of HIV}

The system (8) can be written as

$$
\left.\begin{array}{l}
\dot{x}=-a_{1} P_{j}-a_{2} X_{j}+a_{3}, \\
\dot{y}=a_{1} P_{j}-a_{4} Y_{j} .
\end{array}\right\}
$$

where

$$
\begin{aligned}
\Delta x_{j} & =x\left(t_{j}\right)-x\left(t_{0}\right), \Delta y_{j}=y\left(t_{j}\right)-y\left(t_{0}\right), \\
P_{j} & =\int_{0}^{t_{j}} \frac{x(t) y(t)}{x(t)+y(t)} d t, \\
X_{j} & =\int_{0}^{t_{j}} x(t) d t \text { and } Y_{j}=\int_{0}^{t_{j}} y(t) d t .
\end{aligned}
$$

The system (30) can be written in the form (3) with

$$
\mathbb{A}=\left(\begin{array}{cccc}
-P_{1} & -X_{1} & 1 & 0 \\
-P_{1} & -X_{2} & 1 & 0 \\
\vdots & \vdots & \vdots & \vdots \\
P_{1} & 0 & 0 & -Y_{1} \\
P_{1} & 0 & 0 & -Y_{2} \\
\vdots & \vdots & \vdots & \vdots
\end{array}\right)
$$

and

$$
\mathbf{h}=\left(\Delta x_{1}, \Delta x_{2}, \ldots, \Delta y_{1}, \Delta y_{2}, \ldots\right)^{\top}
$$

\section{NUMERICAL EXTRACTION OF MODEL PARAMETERS}

In order to illustrate the effectiveness of the method the parameter identification of the system (8) is presented. Using parameter values from Gumel [4], we generate points of solutions of the system (8) by the adaptive Runge-Kutta method. A mathematical software Mathcad was used for the adaptive Runge-Kutta method and for the integration by quadratue rules. These solutions are then perturbed by a normal distribution with mean $\bar{x}$ and standard deviation $\delta=0.5$ and further taken as "experimental data".

Furthermore, system (8) is studied in the context of the Gauteng Province, South Africa. Data used in the numerical simulation is obtained from the Acturial Society of South Africa (ASSA) (see [2]) HIV prevalence estimates. The data is compiled from the population sensus, antenatal survey and registered deaths [2]. The ASSA 2003 tables gives the population $N$ and the number infected with HIV $y$. From the relationship

$$
N=x+y,
$$

we obtain the susceptible population $x$ as

$$
x=N-y .
$$

The results given in Table 2 below, show a comparison of the parameters used (from Gumel [4]), the estimated parameters and the percentage error given by $\|\alpha-\tilde{\alpha}\| /\|\alpha\| \times 100$. 
A.N. Pete et al., Determination of parameters for Cauchy's problem ...

Table 2: Problem 4: The parameter estimates and errors.

\begin{tabular}{cccc}
\hline Parameter & Actual value & Estimated value & $\%$ Error \\
& $\alpha$ & $\tilde{\alpha}$ & \\
\hline$a_{1}$ & 0.24 & 0.2404 & 0.375 \\
$a_{2}$ & 0.03125 & 0.0313 & 0.16 \\
$a_{3}$ & 2000 & 2000 & 0.000 \\
$a_{4}$ & 0.531 & 0.532 & 0.16 \\
\hline
\end{tabular}

Applying the parameter estimation method in the case of the transmission dynamics of HIV in the Gauteng Province, South Africa the following estimates are obtained:

$$
\left(\begin{array}{l}
a_{1} \\
a_{2} \\
a_{3} \\
a_{4}
\end{array}\right)=\left(\begin{array}{c}
0.393 \\
0.049 \\
565143 \\
0.149
\end{array}\right) .
$$

Figures 1 and 2 below show the comparison of the estimated solutions with the "experimental data" and the absolute error in the decimal logarithmic scale. The initial value problem was solved with the new coefficients and old initial conditions.
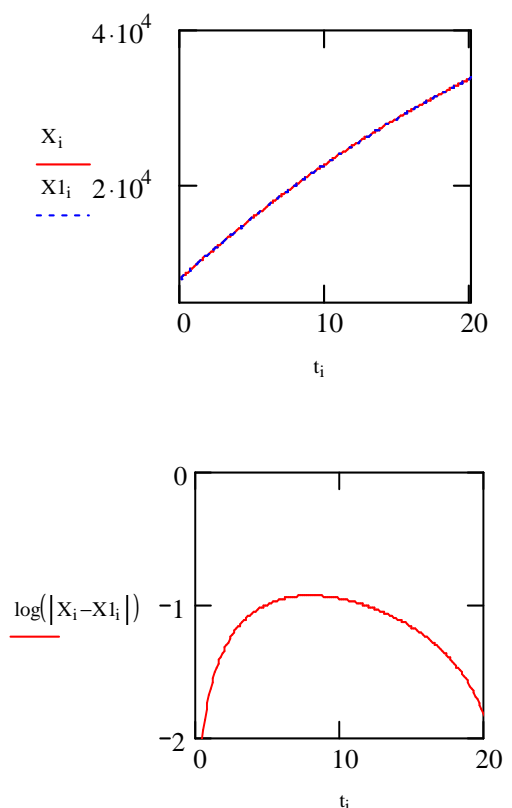

Fig. 1. Comparison of the estimated solutions, $X_{i}$, with the "experimental data" and the absolute error in the decimal logarithmic scale.
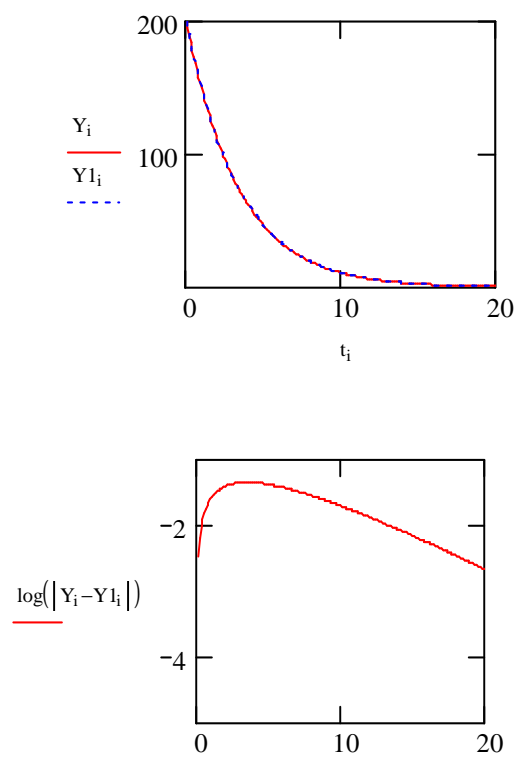

Fig. 2. Comparison of the estimated solutions, $Y_{i}$, with the "experimental data" and the absolute error in the decimal logarithmic scale.

From the comparison of the parameters in Table 2, Figures 1 and 2, it can be seen that the estimated parameters are close enough to the actual values. The estimated parameters of dynamic HIV mathematical models for the Gauteng Province are all nonnegative and also in the expected ranges.

\section{CONCLUSiON AND SUGGESTIONS FOR FURTHER RESEARCH}

A method for estimating parameters of dynamic mathematical models given some information about unknown trajectories at some time, by Shatalov, Fedotov and Joubert [7], was applied to problems from Biology and Ecology. In Problem 2 where the initial value was assumed to be unknown with accuracy, the method was applied to find this value. To illustrate the efficiency of the method, a numerical example for the model that monitors the dynamics of HIV was presented. The estimated parameters for the artificially generated data are close enough to the actual parameter values and for the Gauteng Province the estimated parameters 
A.N. Pete et al., Determination of parameters for Cauchy's problem ...

are in the expected ranges.

The method for estimating parameters values could be improved by incorporating a suitable penalty term that minimizes the error caused by numerical quadrature and high observational noise levels in the real data. An improvement of the method will be investigated in future studies.

\section{REFERENCES}

[1] A. D. Bazykin, Nonlinear Dynamics of Interacting Populations, Singapore, World Scientific, 1998.

[2] R. E. Dorrington, L. F. Johnson, D. Brandshaw \& T. Daniel, The demographic impact of HIV/AIDS in South Africa: National and provincial indicators, Cape Town: Centre for Acturial Research, South African Medical Research Council and Actuarial Society of South Africa, 2006.
[3] S. S. Dragomir \& I. Fedotov, A Gruss type inequality for mapping abounded variation and applications for numerical analysis, Nonlinear Functions. Anal., Appl., $6(3)$ : $425-433,2001$.

[4] A. B. Gumel, A competitive numerical method for a chemotherapy model of two HIV subtypes, Applied Mathematics and Computations, 131 : 329 - 337, 2002.

[5] C. L. Lawson \& R. J. Hanson, Solving Least Square Problems, New Jersey, America: Prentice-Hall, 1974.

[6] M. Shatalov \& I. Fedotov, On identification of dynamic systems parameters from experimental data, RGMIA, Victoria University, 10(1,2) : 106 - 116, 2007.

[7] M. Shatalov,I. Fedotov \& S. V. Joubert, A novel method of interpolation and extrapolation of functions by a linear initial value problem, Buffelsfontein TIME 2008 Peerreview Conference Procedings, 22 - 26 September, 2008. 